\title{
Implementation of scada system to the central liquor factory
}

\author{
José Enrique García Arteaga ${ }^{1}$, Samy Brito Barroso ${ }^{2}$, Liodán Valdivia Coca ${ }^{3}$, Orlando \\ Urquijo Pascual ${ }^{4}$, Lisdán Herrera García ${ }^{5}$
}

\author{
1,2,3,4,5 UCLV, Cuba, Calle 9na. \# 411, e/ 4ta. y 6ta., Rpto Universitario, Santa Clara V.C, Cuba.
}

Email: arteaga@uclv.edu.cu, samyb@uclv.edu.cu,coca@vc.copextel.com.cu, orlandop@uclv.edu.cu, 1herrera@pdvcupetsa.cu

Received: November $28^{\text {th }}, 2017$.

Accepted: February $02^{\text {th }}, 2018$.

Published: March $30^{\text {th }}, 2018$.

Copyright (C2016 by authors and Institute of Technology Galileo of Amazon (ITEGAM).

This work is licensed under the Creative Commons Attribution International

License (CC BY 4.0).

http://creativecommons.org/licenses/by/4.0/ (c) (1) (2)(2) Open Acten:

\begin{abstract}
The implementation of a SCADA application on the Central Liquor Factory "Agustin Rodriguez Mena" belonging to the Company Cubaron has eliminated the low interconnectivity between machines installed and it has avoided the need to establish control strict on ingredients used in the elaboration of the final product. After completion of preliminary studies on the particularities of the industry and instrumentation contained therein, the identification of main control variables in the PLC has taken place and modifications were made in the PLC program for the variables that would be reflected in the SCADA and OPC server for communication. The control and monitoring of the overall process of rum manufacturing will increase efficiency, it will allow for rapid detection of faults in the system and it will make easier the work of operators.
\end{abstract}

Keywords: Label, OPC, PLC, SCADA, supervise.

\section{Implementación del sistema scada en la fábrica central de licores}

\begin{abstract}
RESUMEN
La realización de una aplicación scada en la Ronera central "Agustín Rodríguez Mena" del Municipio de Santo Domingo perteneciente a la Empresa Cubaron elimina la baja interconectividad entre las máquinas instaladas y la necesidad de establecer un control estricto en la materia prima utilizada en la elaboración del producto final. Finalizados los estudios preliminares sobre las particularidades de la industria y la instrumentación contenida en ella; se procedió a la identificación en el PLC de las principales variables a controlar y se realizaron modificaciones en el programa del mismo para obtener las variables que serían reflejadas en el SCADA y en el servidor opc para la comunicación. El control y la supervisión de todo el proceso de fabricación del ron, aumentará la eficiencia, permitirá una rápida detección de fallas en el sistema y hará más fácil el trabajo de los operadores.
\end{abstract}

Palabras Claves: enjuagadora, etiquetadora, OPC, PLC, SCADA, supervisión.

\section{INTRODUCCIÓN}

Con el paso del tiempo la tecnología há evolucionado hasta desarrollar varios sistemas y arquitecturas para el control, supervisión y la adquisición de datos en todo tipo de industria o proceso. Los sistemas SCADA aportan, fiabilidad, flexibilidad, aumento de la producción y mejoras em las condiciones d operación de las plantas. Además, disminuyen las pérdidas, los accidentes por concepto de errores humanos, aumentan la eficiencia y la calidad del producto con una disminución de los costos de producción.

Actualmente es posible acceder e intercambiar gran cantidad de datos de producción entre diferentes niveles de la fábrica, por ejemplo; en presencia de una adecuada arquitectura de red, un operador en el nivel administrativo podría ganar acceso a los datos del nivel de campo o control y usar los mismos em algún tipo de procesamiento en línea para generar diagramas de tendencia en tiempo real, útiles para predecir la cantidad de suministros requeridos para encarar un periodo de producción definido. Obviamente, estos datos se pueden almacenar em una base de datos histórica, lista para ser usada em procedimientos de análisis de pos procesamiento sucesivo [1][2].

La utilización de SCADA en la industria há originado una tendencia creciente [3-6] y la industria licorera no se ha quedado atrás [7], la Planta COPLAN y Planta "El Tiro" de la Firma Osbourne (La empresa S.G.A. Distribuciones Especializadas, S.L. ha desarrollado el sistema automático de control de tres líneas de 
embasado en la planta embotelladora "El Tiro" perteneciente a la empresa Osbourne.)

En Cuba, se han comenzado a introducir em algunas plantas como ejemplo está la utilización de um SCADA en la Ronera de San José, en la provincia de la Habana, que se dedica a la producción de rones tintos de alta calidad.

En la Ronera Central, se producen distintos tipos de rones (Havana Club, Cubay en sus variedades) para el mercado nacional y extranjero. La decisión de realizar este proyecto se originó porque en la fábrica no se explotaban las potencialidades con las que cuenta; la gestión, la comunicación a nível empresarial y la supervisión de la producción se encontraban en un nivel muy bajo, además de no existir visualización del proceso productivo. También se hacía necesario establecer un control riguroso de la materia prima utilizada para la elaboración del producto, la cual es de importación y resulta muy costoso adquirirla.

\section{METODOLOGÍA}

\section{II.1 FUNCIONALIDADES DE UN SISTEMA SUPERVISORIO}

En la actualidad los proveedores de los SCADA diseñan sistemas que son pensados para resolver las necesidades de muchas industrias, con módulos de software específicos disponibles para proporcionar las capacidades requeridas comúnmente. Los sistemas SCADA constituyen parte integral de la estructura de dirección y gerencia de cualquier empresa. Estos sistemas no son vistos por la gerencia como herramientas operacionales de supervisión y control, sino como un importante recurso de información corporativa sin el cual sería imposible administrar correctamente una empresa.

Los sistemas SCADA utilizan la computadora y tecnologías de comunicación para automatizar el monitoreo y el control de procesos industriales. Estos sistemas son partes integrales de la mayoría de los ambientes industriales complejos o muy geográficamente dispersos, ya que puede recoger la información de una gran cantidad de fuentes muy rápidamente, y la presentan a un operador de uma forma amigable. Estos sistemas mejoran la eficácia del proceso de monitoreo y control proporcionando la información oportuna para poder tomar decisiones operacionales rápidas [8].

El objetivo fundamental de un sistema supervisório es brindar los medios al operador humano para controlar y comandar un proceso altamente automatizado. Así, la supervisión de los processos industriales incluyen un conjunto de tareas que ayudan a controlar los procesos y supervisar sus operaciones [9].

Los SCADA se comportan como un sistema industrial de mediciones y control que consiste según el criterio de distintos especialistas de uma computadora principal o máster (generalmente llamada Estación Principal, o MTU); una o más unidades de control obteniendo datos de campo (comúnmente llamadas estaciones remotas, o RTU's), sistema de comunicación y la interface hombre máquina [2][9].

A través de los mismos se puede conocer el estado de una instalación, centralizando la información de los emplazamientos remotos en uno o varios puestos de control. Los equipos de control situados en las estaciones analizan los parâmetros más importantes recogiendo los valores aportados por los diferentes sensores. Cuando se identifica uma situación de alerta estos equipos realizan la operación adecuada y advierten del mismo al puesto de control, desde donde se procesa la información y se genera de forma automática la señal de mando apropiada. Desde el puesto central se puede obtener, en tiempo real, cualquier información relativa a las estaciones.
Además de gestionar alarmas y de capturar datos, los sistemas SCADA permiten generar planes de mantenimiento y eficaces procedimientos de actuación para los operadores. Estos facilitan el trabajo del personal de mantenimiento permitiendo automatizar procesos de muy alta complejidad.

En la literatura científica se encuentran diversas tendencias en el uso de sistemas SCADA entre ellas se puede mencionar: S. A. Avlonitis \& al [10], han presentado la estructura e instalación de un sistema SCADA flexible y de bajo costo que incluye una PC con su software e interfaces ordinarias que operan el sistema.

Patel \& al. [11], han presentado un sistema SCADA que permite la comunicación con vários dispositivos $\mathrm{E} / \mathrm{S}$ en los sistemas de energia renovables y componentes de instalaciones de prueba RESLab. Este sistema SCADA difiere de los tradicionales sistemas SCADA en que se apoya en um entorno operativo que cambia continuamente em función de la prueba a realizar; el mismo se basa em el concepto de tener un servidor Máster de E / $\mathrm{S}$ y múltiples sistemas informáticos como clientes. Los autores han descrito las principales características y ventajas de este sistema SCADA dinámico, las conexiones de los dispositivos de campo diferentes al servidor máster de E/S, los servidores de dispositivos y el software de múltiples funciones utilizadas en el sistema.

Presentan un trabajo sobre la adquisición de datos de tiempo real y la evaluación de control avanzado utilizando la plataforma de código abierto científica Scilab, se discute en el mismo la implementación y visualización de datos en línea com la caja de herramientas Scicos y la utilización de la tecnología OPC [12]. La viabilidad y eficacia de la metodología propuesta se demostró por medio de la aplicación de controladores difusos en un proceso de precipitación de la enzima bromelina instrumentado con dispositivos de la Fundación Fieldbus. Los resultados confirmaron Scilab / Scicos adecuado para HMI y sistemas de control aplicados en pequenas aplicaciones industriales.

Abordan en su trabajo principalmente el entorno de seguridad único y los problemas inherentes a la red SCADA radial de los sistemas de energía eléctrica. El enfoque esta dirigido a la utilización del método de cifrado simétrico, en la mayor parte del trabajo se limita a la gestión de claves de cifrado y proporcionar una solución para el período óptimo de distribución de claves [13].

Ha desarrollado la idea de que los sistemas SCADA están siendo rapidamente integradas con redes corporativas, pero las ramificaciones de una violación SCADA son mucho más preocupante que la interrupción de la producción [14].

Aydogmus [15], ha presentado un sistema SCADA a través de PLC para un sistema de control de nível de fluido con un controlador borroso. Para este fin, se ha reunido un conjunto del control de nivel de líquido y el PLC. El PLC utilizado no tiene software o módulo borroso, para el mismo ha sido desarrollado um algoritmo borroso de tipo Sugeno para completar el estudio.

El objetivo principal de este trabajo es presentar una aplicación que se ha realizado sin software/módulo de controlador de lógica difusa aplicando este tipo de control.

\section{II.2 METODOLOGÍA DE ANÁLISIS DEL SISTEMA SCADA}

La línea de producción a la cual se le realizará la supervisión se encuentra situada en un espacio relativamente pequeño. Está conformada por cuatro etapas:

Primera etapa: área de descarga, en la cual se reciben los pallets de botellas, se verifica que sean las indicadas para la 
producción que se está desarrollando en ese momento y se incorporan a la estera transportadora.

Segunda etapa: se desarrolla en la máquina denominada Monobloque, en la cual se realizan cuatro operaciones. Primero ocurre el lavado de las botellas con agua previamente tratada com cierto grado de alcohol, para evitar afectar la calidad del producto final. Luego se realiza el llenado de las botellas en la máquina llenadora para en los últimos dos pasos, insertarles los tapones de presión y de rosca por ese orden.

Tercera etapa: se realiza el proceso de etiquetado donde se le adhieren a la botella la etiqueta, contra etiqueta, collarín y sello de calidad.

Cuarta etapa: se encarga de la confección de las cajas y del llenado de las mismas para su posterior traslado. El movimiento de las botellas en todo el processo se realiza a través de esteras transportadoras. La figura 1 muestra el flujo de producción de la línea.

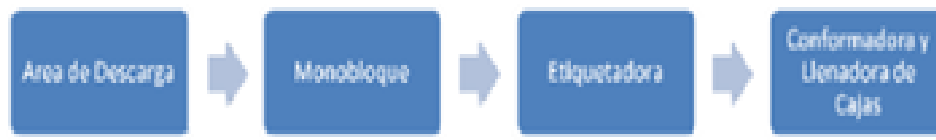

Figura 1: Línea de producción.

Fuente: Los Autores, (2018).

El principal problema que presenta la filial de Cuba Ron SA. en el centro del país es el control sobre la materia prima y la producción, ya que no se conoce con certeza el lugar donde ocurren las ilegalidades y tampoco existe la posibilidad de realizar reportes de producción con datos reales. Además, el nivel de visualización del proceso es mínimo, la comunicación a nivel empresarial no se encuentra implementada y aunque la línea de producción trabaja de forma automática, no existe conectividad entre las máquinas que intervienen en el proceso de embotellado.

Con el objetivo de interconectar las máquinas de la línea se realizaron búsquedas en manuales y planos eléctricos de las mismas; no se obtuvo una solución razonable. Además, se definieron y recolectaron las variables deseadas del proceso, muchas de las cuales están siendo usadas por los PLC's que controlan la línea y se encuentran almacenadas em sus respectivas memorias.

Después de este estudio se propuso implementar un sistema supervisor para poner fin a las deficiências existentes en dicha empresa. Para la implementación del sistema era necesario obtener las principales variables del proceso y para esto se tomó la opción de no modificar el funcionamiento de la línea, pues esta no presentaba dificultades.

La implementación de un sistema SCADA para la supervisión y control de la línea de fabricación, permitirá la obtención de los registros históricos de las variables; el conteo de etiquetas, las contraetiquetas, los sellos y las botellas que entran y salen de la línea, la generación de alarmas, la detección de fallas y averías eficazmente, entre otras soluciones, permitiendo resolver la problemática de dicha industria.

En la línea de fabricación donde se desarrolla este proyecto no existe similitud entre la tecnología y maquinaria instalada, puesto que la etiquetadora y el mono bloque pertenecen a la OMROM y la conformadora y empacadora a la Siemens. En tal caso es necesario estandarizar la comunicación entre los distintos dispositivos de campo (PLC's) y el sistema supervisor utilizando un servidor OPC.

En la actualidad los fabricantes de sistemas SCADA no encuentran en la adopción de OPC uma opción, es simplemente una necesidad por lo que su uso se ha generalizado rápidamente
[16]. En el proyecto se ha utilizado el KEPServerEx 4.0 de Kepware's como servidor OPC.

Este software permite una rápida configuración em la comunicación con los sistemas de control a través de una larga lista de drivers de dispositivos disponibles, también provee una interfaz amigable al usuario que ha garantizado su adopción por parte del personal de planta sin rechazo.

La aplicación desarrollada en el software KEPServerEX 4.0 ha facilitado la comunicación entre las diferentes máquinas del proceso, logrando esto con total transparencia para el SCADA y el usuario, siendo el sistema supervisor un cliente OPC de la aplicación implementada.

Con el paso del tiempo, nuestro país ha llevado a cabo la realización de diferentes proyectos de automatización incorporando sistemas de control y supervisión. El desarrollo en el estudio de estas herramientas ha hecho posible la ejecución y montaje de estos sistemas por las diferentes empresas que se encargan de esta labor, como Copextel, CEDAI, entre otras. En su gran mayoría han sido implementados con el software Movicon, proporcionado por Progea, el cual se ha convertido en una herramienta fundamental para los técnicos cubanos por las prestaciones que este brinda.

En el proyecto desarrollado se ha utilizado el Movicon X2 como software de desarrollo del sistema SCADA, constituye una herramienta para las compañías que trabajan en el campo de la automatización y el control de procesos y edifícios inteligentes. Permite la adquisición de datos a través de su comunicación con el PLC, red y bus de campo, así como la configuración de herramientas y sensores. Los datos adquiridos se coleccionan dentro de una base de datos en tiempo real (RTDB) para luego estar disponibles por los objetos y recursos para crear de forma animada, sinópticos, alarmas, recetas, gráficos y reportes.

Los PLC que controlan la maquina etiquetadora (SYSMAC CPM2A) y el mono bloque (SYSMAC CQM1H) pertenecen a la firma OMROM, para mejorar el funcionamiento del mismo y mostrar en el SCADA datos necesarios en el control de la materia prima, se procedió al estudio y mejora del programa de cada PLC. La herramienta de software utilizada para la programación de las secciones de programa necesarias para cumplimentar los objetivos, es el CXProgrammer versión 3.0. CX-Programmer es uma herramienta de programación para la creación, prueba y mantenimiento de programas asociados con PLCs de OMROM cuyas series sean CS/CJ, CV y C. Proporciona las instalaciones de los dispositivos PLC, los modos de comunicación con los PLC de OMROM y permite la configuración con diferentes tipos de red.

\section{II.3 DESARROLLO DEL SCADA}

Como primer paso se realizó el estudio del proceso productivo, así como del funcionamiento y las características del Movicon X2. Se tomaron fotos de las máquinas y de las líneas de producción em general, con el objetivo de que al realizar la interfaz gráfica (HMI) esta se asemejara lo más posible al proceso y los operarios se adaptaran al sistema com mayor facilidad. Fue presentado por parte de la empresa un bosquejo de las necesidades de los operarios y técnicos en toda la línea de producción. A raíz de esto se analizaron cuáles eran las variables de mayor importancia a supervisar y controlar en cada máquina y en toda la línea de producción. Por su importancia en el la línea de fabricación se concentran los esfuerzos en las etapas dos y tres del proceso.

Para la segunda etapa se decidió reflejar en uma pantalla principal llamada "Monobloque" (Figura 2), (con un ambiente 
muy parecido a la realidad de la máquina), la mayor cantidad de variables posibles que manejaba esta máquina. Se tuvo en cuenta un orden de prioridad, de esta forma se reflejaban las variables más importantes y no se carga la pantalla com demasiada información. Se crearon otras pantallas donde el operador tiene la posibilidad de acceder a datos más detallados del proceso, como es el caso de las dedicadas a las Alarmas, Enjuagadora, Térmicos, Conteo de Botellas, etc. Además se creó una barra de "Menú", visible en la parte superior de la pantalla "Monobloque" en modo de ejecución, desde donde se puede acceder a la información que se necessite manejando las propiedades dinámicas de estos.

La figura 2 muestra el sinóptico que el operador estará visualizando la mayor parte del tiempo. Constituye la pantalla que más información ofrece. Muestra los principales elementos de la máquina, como el estado del Motor Principal y el de Cinta transportadora, la velocidad con que opera la misma, además del reconocimiento de las principales paradas y reducciones de velocidad. Se muestran también datos particulares de las diferentes secciones que integran el Monobloque y se introdujo a petición de los operadores un botón de emergencia, el cual fue necesario programar en el PLC. Desde esta pantalla es posible acceder a los demás sinópticos que se encuentran disponibles gracias a un Menú que se divisa en la parte superior de la misma. También se muestra la fecha y hora, como un requisito indispensable.

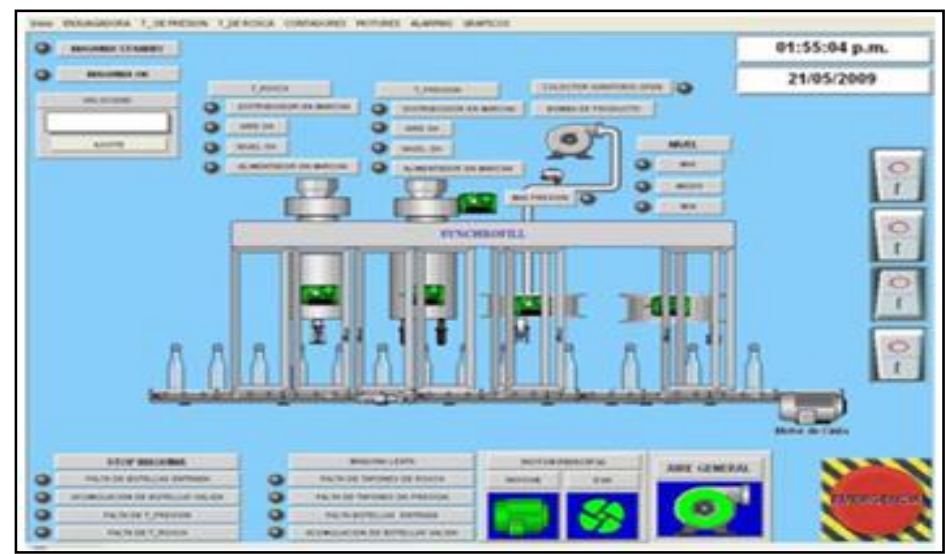

Figura 2: Monobloque.

Fuente: Los Autores, (2018).

Dentro del Monobloque a la máquina de la "Enjuagadora" (Figura 3) se dedicó un sinóptico recreando algunos de los eventos que pueden ocurrir en esta parte del Monobloque como la falta de presión en los diferentes tratamientos de enjuague, la fricción en las estrellas de entrada y de salida, el correcto funcionamiento de la máquina, el estado de la bomba de tratamiento y la alarma de contraguía sinfín en la Enjuagadora.

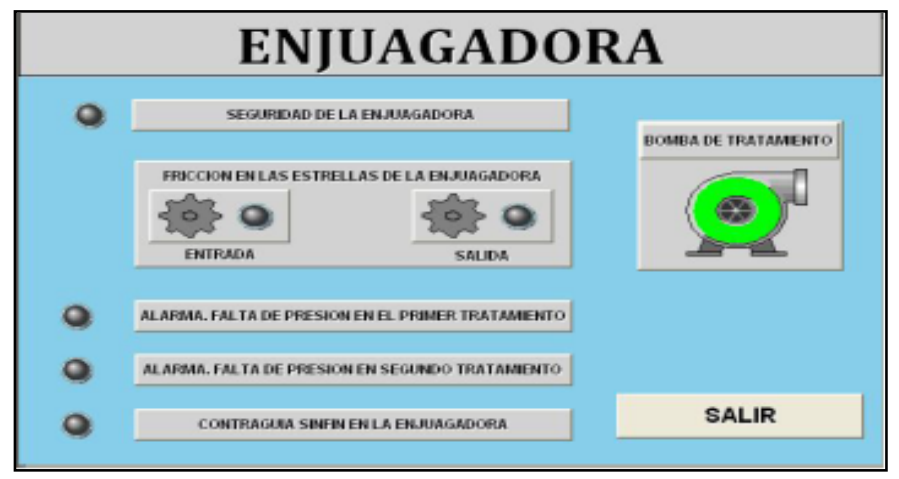

Figura 3: Enjuagadora en el monobloque.

Fuente: Los Autores, (2018).
A los distintos contadores se dedicó también su interfaz, separándolos en Conteo de Botellas y Mermas (Figuras 4 y 5).

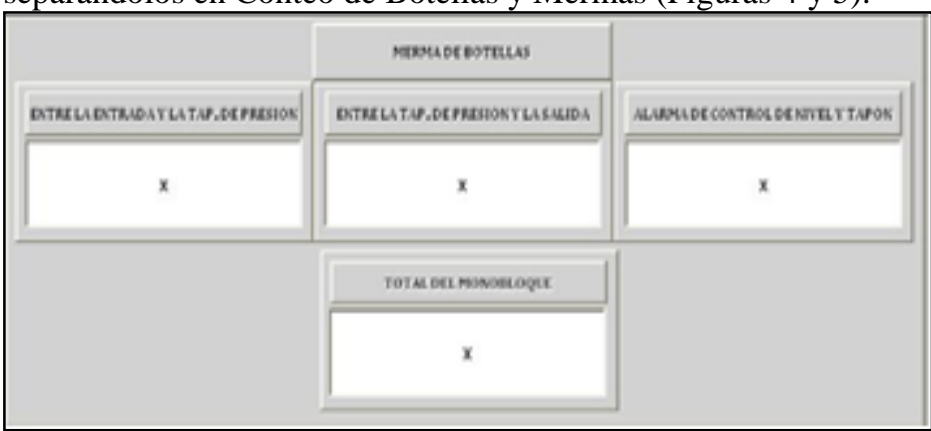

Figura 4: Conteo de botellas en el monobloque.

Fuente: Los Autores, (2018).

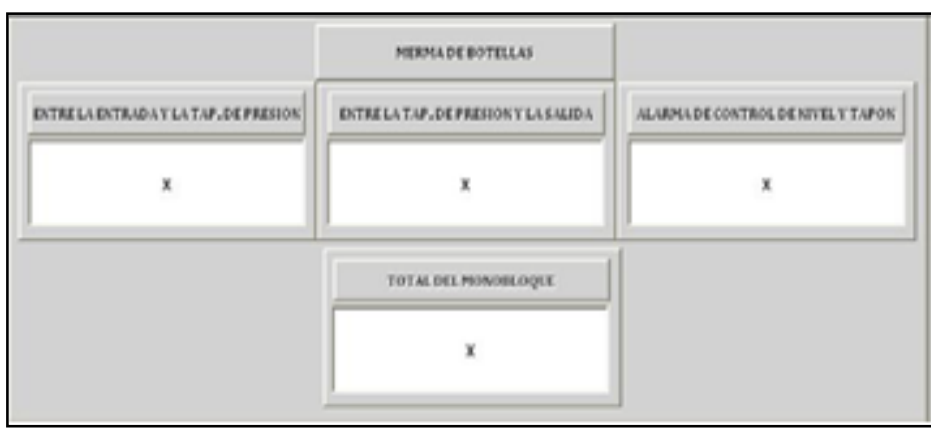

Figura 5: Conteo de mermas en el monobloque.

Fuente: Los Autores, (2018).

Para la tercera etapa en la máquina etiquetadora se creó una pantalla principal llamada "Etiquetadora" (Figura 6), desde donde el operario supervisará de manera general el proceso de etiquetado, existen otras ocho pantallas dentro de esta con las demás especificaciones de dicho proceso. Este sinóptico muestra la máquina etiquetadora, las tres máquinas alimentadoras del juego de etiquetas (collarín, etiquetas, contraetiquetas) con sus motores, dos casillas de edición con la hora (en modo solo lectura) y fecha (asociadas a variables del sistema), las esteras transportadoras, un símbolo destinado a alertar al trabajador que existe un fallo en la comunicación y el botón de emergencia, con el cual se manda a detener el sistema de forma imediata ante cualquier situación que el operador considere anormal.

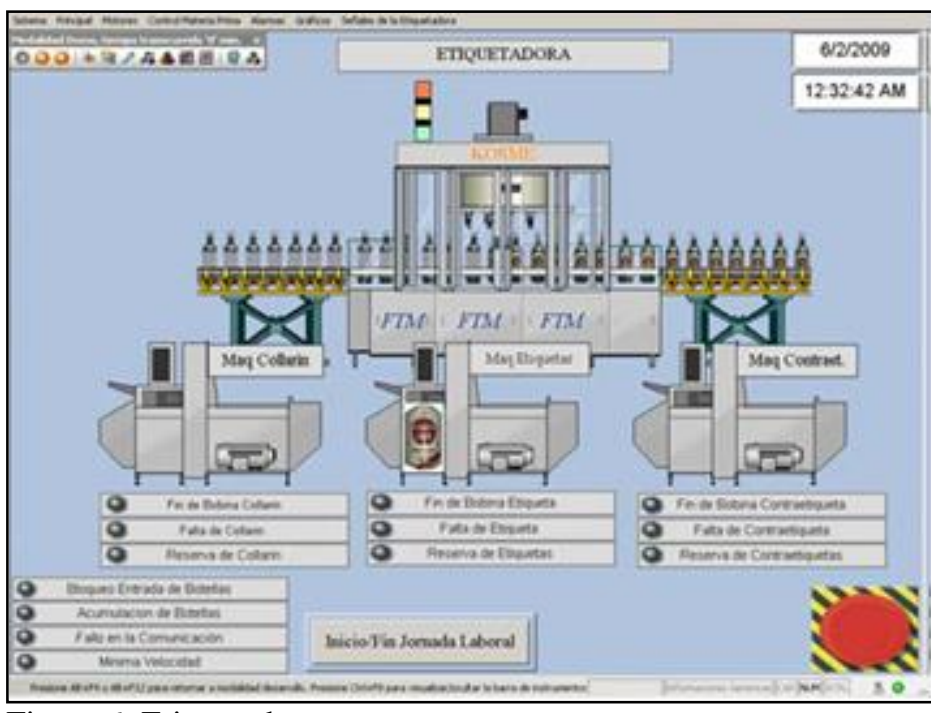

Figura 6: Etiquetadora.

Fuente: Los Autores, (2018). 
En el proceso de etiquetado se creó una pantalla (Figura 7 que nos da el índice de botellas de entrad contra el juego d etiquetas. Es decir muestra e control de la producción (botella etiquetadas cantidad de cajas) con respecto a materia prim (collarín, etiqueta, contraetiqueta). En este sinóptic se muestran los respectivos contadores de botellas la entrada, el número d botellas etiquetada correctamente así como incorrectamente representados por casillas de edición. Los botones d reset para poner en cero los contadores ante mencionados. Además se muestra una casilla d edición para representar el tiempo perdido y u pulsador de reset (tiempo sin trabajar la máquina).

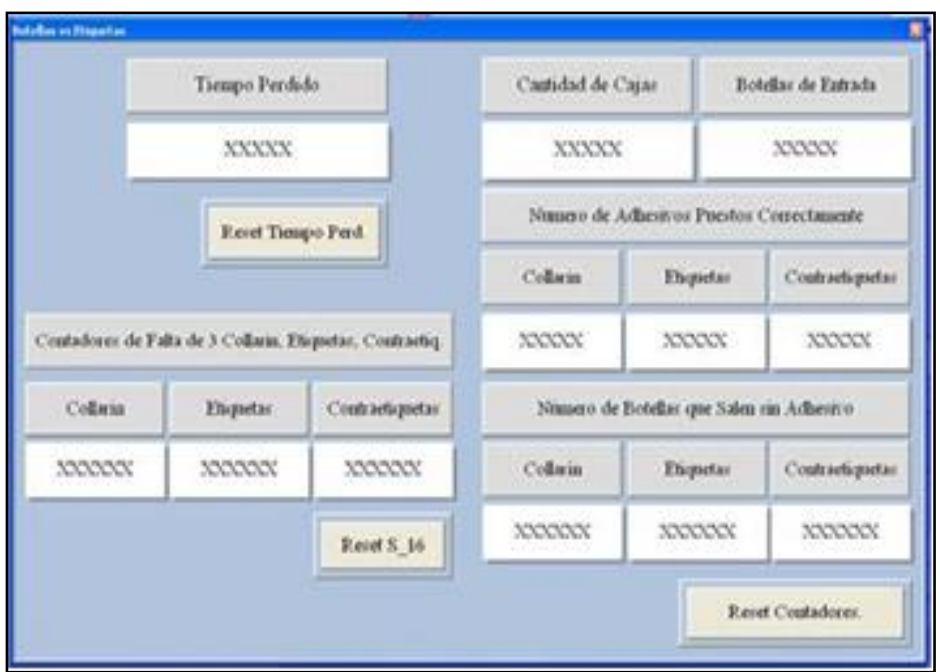

Figura 7: Pantalla de control de la materia prima y de la producción.

Fuente: Los Autores, (2018).

Otra pantalla de gran ayuda para la supervisión e la dedicada al tratamiento de las alarmas (figura 8). La misma aporta datos importantes como el estado de la alarma, el momento en que ocurrió, el tiempo de duración, una breve descripción y el lugar de origen. Consta con propiedades de sonido, para llamar la atención del operador con mayor rapidez.

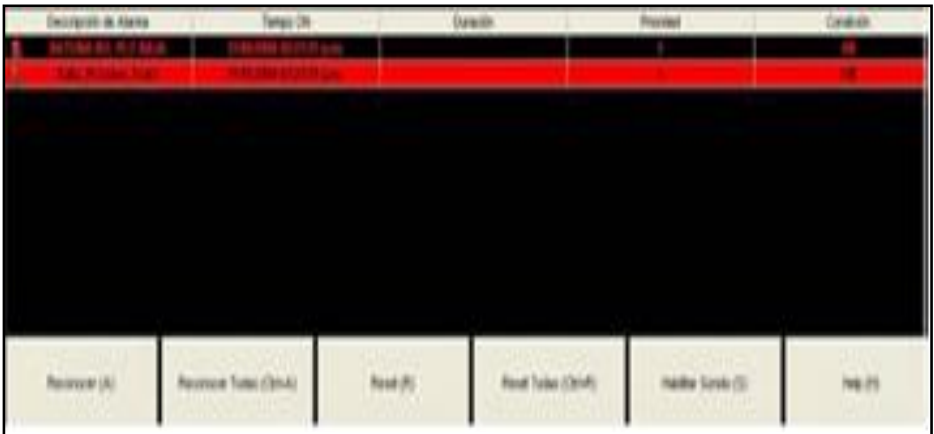

Figura 8: Pantalla de alarmas.

Fuente: Los Autores, (2018).

Este sinóptico se activará de manera automática en la computadora dependiendo de la prioridad que se le otorgue a la alarma. Eventos de envergadura como las averías en los motores y la falta de comunicación con el PLC fueron categorizados de prioridad uno, y los mismos activaran de manera inmediata la pantalla para que el operador realice la acción que estime conveniente en el menor tiempo posible. Se creó además, la pantalla de Data Logger (Figura 9), en la cual se podrá conocer el estado de las variables que estén asociadas a esta de acuerdo con el tiempo de refrescamiento que se programe a la variable.

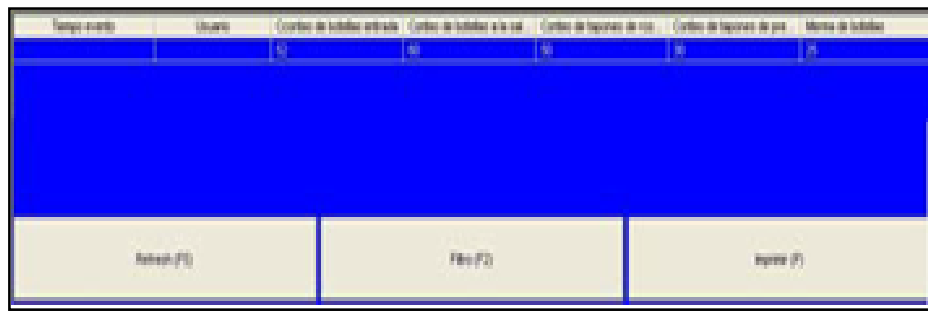

Figura 9: Pantalla del data loger.

Fuente: Los Autores, (2018).

Se implementaron además pantallas gráficas de reportes históricos con datos como la producción, el tiempo perdido, la merma de materia prima (botellas, juego de etiquetas etc.) previamente definidos en los data loggers. Además presenta en la parte inferior botones que facilitan la revisión y análisis de los datos históricos (valores máximos y mínimos) así como la impresión de estos

Para la supervisión de la planta era necesaria la inclusión en la programación de los PLC, de algunas líneas de código que garantizaran cumplir con el objetivo planteado. Como resultado del trabajo, quedaron implementados cambios que permitieron una disminución del tiempo de ejecución del programa, la obtención de las variables más importantes que serían visualizadas en el SCADA y el conteo de las materias primas que entran y salen de las diferentes maquinas, lo cual no se llevaba a cabo anteriormente.

\section{RESULTADOS Y DISCUSIÓN}

En este artículo, se han presentado las características y funcionalidades del sistema SCADA realizado para la línea de embotellado y etiquetado de la empresa "Agustín Rodriguez Mena", el mismo cumple con los requisitos deseados de supervisión y control y mejora la calidad de la información que se obtiene del proceso lo cual resulta beneficioso desde el punto de vista económico. Los segmentos de programa y los nuevas variables adicionadas a los PLC, ayudaron a la realización del sistema SCADA y mejoraron el funcionamiento de la línea de producción. Se incrementó el nivel de información, control y supervisión de la producción y la matéria prima en toda la línea de producción. El Movicon X2 demostró ser una herramienta que ofrece la posibilidad de realizar potentes y compactos sistemas de supervisión y control con agradables interfaces HMI.

Para futuras etapas se prevé utilizar el sistema de reportes "Crystal Report" para generar los reportes em la empresa. También se propone la exportación de la aplicación SCADA a un servidor web para facilitar la gestión operativa y empresarial.

Actualmente se realizan propuestas preliminares para extender las aplicaciones SCADA a otras ramas de la economía.

\section{REFERENCIAS}

[1] Adamo, F., Attivissimo, F., Cavone, G., Giaquinto, N., "SCADA / HMI Systems in Advanced Educational Courses", IEEE Transactions on Instrumentation and Measurement, Vol. 56, (1), pp. 4-10, 2007.

[2] Thomas, M. S., Kumar, P., Chandna, V. K., "Design, Development, and Commissioning of a Supervisory Control and Data Acquisition (SCADA) Laboratory for Research and Training", IEEE Transactions on Power Systems, Vol. 19, (3), pp. 1582-1588, 2004. 
[3] Köstner, S., "Control and Operation of the LHCb Readout Boards Using Embedded Microcontrollers and the PVSS II SCADA System", IEEE Nuclear Science Symposium Conference Record, pp. 759-762, 2006.

[4] Ordean, M., Chiorean, D., Rogoz, I., Lehene, C., Stoian, I., Stancel, E., "SCADA Systems - Support for the Maintenance Management of Hydro Power Plants", Automation, Quality and Testing, Robotics, IEEE International Conference, Vol. 1, pp. 238- 242, 2006.

[5] Haijing, Y., Yihan, Y., Dongying, Z., "The Structure and Application of Flexible SCADA", Power Engineering Society General Meeting, pp. 4, 2006.

[6] Giacchini, M., Mastinu, P.F., Gramegna, F., Gadea, A.R., "VPS-Versatile Power Supply Control System", IEEE Transactions on Nuclear Science, Vol. 53, (3), 2006.

[7] Benavides, J. L., "Propuesta de Automatización de una fábrica de licores de Ecuador". Tesis de Maestría, Departamento Automática, Facultad de Ingeniería Eléctrica, Universidad Central "Marta Abreu" de las Villas, Santa Clara, Cuba, 2007.

[8] Rodríguez, A., Loreto, J. L., "Distributed Control Implementation in CVG EDELCA Hydroelectric Power Plants", 2006.

[9] Lakhoua, M.N., "Application of Functional Analysis on a SCADA System of a Thermal Power Plant", Advances in Electrical and Computer Engineering, Vol. 9, (2), pp. 90-98, 2009.

[10] Avlonitis, S.A., Pappas, M., Moutesidis, K., Avlonitis, D., Kouroumbas, K., Vlachakis, N., "PC based SCADA system and additional safety measures for small desalination plants", Desalination 165, pp.165-176, 2004.

[11] Patel M., Cole G. R., Pryor T. L., Wilmota N. A., "Development of a novel SCADA system for laboratory testing", ISA Transactions 43, pp.477-490, 2004.

[12] Costa, T. V., Fileti , A. M., Silva F., "Scilab/Scicos: Na Alternative Tool for Real-Time Monitoring and Advanced Control of Fieldbus Industrial Systems", Computer Aided Chemical Engineering Vol. 27, pp. 1617-1622, 2009.

[13] Kang, D.J., Lee, J.J., Kim, B.H., Hur, D., "Proposal strategies of key management for data encryption in SCADA network of electric power systems", International Journal of Electrical Power \& Energy Systems, 2009.

[14] Munro K., "SCADA - A critical situation", Network Security, Vol. 2008, (1), pp.4-6, 2008.

[15] Aydogmus Z., "Implementation of a fuzzy-based level control using SCADA", article in press, Expert Systems with Applications: An International Journal, Vol. 36, (3), pp 65936597, 2009.

[16] Sahin, C., Bolat, E.D., "Development of remote control and monitoring of web-based distributed OPC system",
Computer Standards \& Interfaces Vol. 31, (5), pp. 984-993, 2009. 